\title{
Macrofungi of oligotrophic Scots pine forests in northern Finland
}

\author{
HENRY VÄRE, ESTERI OHENOJA AND RAUNI OHTONEN
}

\begin{abstract}
VÄre, H., Ohenoja, E. \& Ohtonen, R. 1996: Macrofungi of oligotrophic Scots pine forests in northern Finland. - Karstenia 36:1-18. Helsinki. ISSN 0453-3402

As part of the Lapland Forest Damage Project, permanent sampling plots in oligotrophic dry pine forests with sparse birch or spruce undergrowth were established in Finnish Lapland. One additional site was established in northern Norway. Sporocarps were collected from 14 plots in autumns 1991-1994. All together 207 fungus species were identified, 167 of them stipitate fungi and 40 corticioid or polyporoid species. Seventy-two fungus species were found at only one site, and 44 species at two or three sites. The remaining 91 species (47\%), which were found at more than three sites, may be considered as common in the northern boreal pine forests of eastern Fennoscandia. Fifteen of these species were not especially associated with pine. Fifty-five per cent of all species were mycorrhizal, but as much as 70\% of the common ones. In total, about 152 species were associated with pine. Ninety of these were mycorrhizal species, eight of them perhaps also mycorrhizal with birch or spruce; 44 were wood decomposers and 18 humicular species, decomposing mainly pine needles; 14 saprophytic species lived amongst mosses. The ectomycorrhizal genus Cortinarius was overwhelmingly most rich in species, with about 35 distinguished taxa. Of humicular saprophytic species $75 \%$ belonged to the family Tricholomataceae. The 58 wood decomposers mostly belonged to Corticiaceae and Polyporaceae. Only one basidiolichen, one coprophilous and one mycophilous species were found. The average annual yield of sporocarps was $4600 \mathrm{~g} \mathrm{dw} \mathrm{ha}^{-1}$. The variation from one year to the next was marked, with the most productive year being 1993 (9685 g $\left.\mathrm{dw} \mathrm{ha}^{-1}\right)$ and the poorest $1994\left(1115 \mathrm{~g} \mathrm{dw} \mathrm{ha}^{-1}\right)$. Four species, Suillus variegatus, Lactarius rufus, Russula decolorans and $R$. paludosa, were responsible for $75 \%$ of the total yield in the best year. All of them are mycorrhizal and edible. The average annual yield of edible species was $3170 \mathrm{~g} \mathrm{dw} \mathrm{ha}^{-1}$, nearly $70 \%$ of the total yield. Four genera produced $85 \%$ of the yield, namely Cortinarius which accounted for $20 \%$, Lactarius for 26\%, Russula for $15 \%$ and Suillus for $27 \%$. The proportion of mycorrhizal fungi was $98 \%$.
\end{abstract}

Key words: Ascomycota, Basidiomycota, boreal pine forests, decomposer, macrofungi, mycorrhiza, saprophytic, yield

Henry Väre, Esteri Ohenoja and Rauni Ohtonen, Department of Biology, University of Oulu, P. O. Box 400, FIN-90571 Oulu, Finland

\section{Introduction}

Dry oligotrophic Scots pine (Pinus sylvestris) forests account for about 3700000 ha (75\%) of all forest in the province of Lapland, northern Finland (Aarne 1994). Our knowledge of the fungal species inhabiting these dry pine forests is scant. Moreover, there are some poorly known genera, rich in species, which are responsible for a large proportion of the fungal yield. The genus Cortinarius is an example (Soop 1993a). Some less abundant genera, such as Hebeloma and Inocybe, are poorly known, too. 
Studies dealing with the yield of macrofungi were recently briefly reviewed in this journal (Salo 1993) and the information will not be repeated. The yield of macrofungi in northern Finland was measured in Scots pine forests between 1976 and 1978 (Ohenoja \& Koistinen 1984), and further south, mainly in the province of Oulu, in 1976-1988 (Ohenoja 1993). These studies serve as a basis for the present, similar investigation in the area.

In the present study macrofungi and their yield in northern Scots pine forests were investigated in the autumns of 1991-1994. Saprophytic and parasitic microfungi were not included, although some of these, such as Exobasidium splendens, occurred at nearly all sites. This article will serve as a basis for forthcoming, more detailed investigations on the composition and structure of macrofungal communities in northern Finland and their relationship to various environmental variables.

Fig. 1. Study sites and their location in northern Finland. 1 = Aska, $2=$ Muonio, $3=$ Raja-Jooseppi, $4=$ Kirakkajärvi, $5=$ Svanvik, $6=$ Angeli, $7=$ Kevo, $8=$ Naruska, $9=$ Tennilä, $10=$ Värriö, 11 Keminmaa, $12=$ Kessi, $13=$ Lokka and $14=$ Kemijärvi. The boundary between the middle and northern boreal zones is according to Ahti et al. (1968).

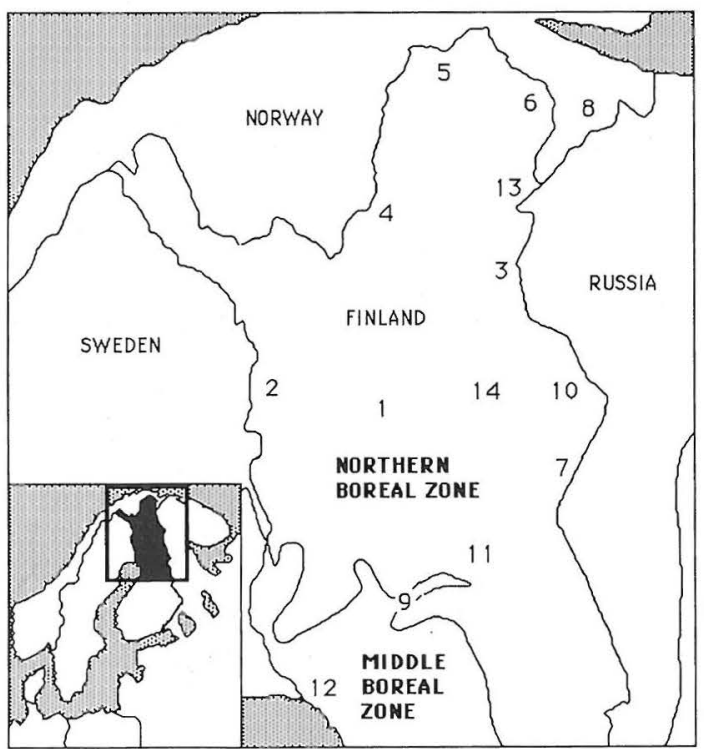

\section{Material and methods}

According to Tuhkanen (1984), the study sites are located in intermediate parts between oceanic and continental sectors in the northern boreal zone, except for the two southernmost sites, which are located in the middle boreal zone (Fig. 1).

The altitude of the study sites varies between 45 and $280 \mathrm{~m}$ above sea level, the mean being $180 \mathrm{~m}$. Only one site, Keminmaa, is under $100 \mathrm{~m}$ a.s.l. The mean annual temperature is $-1.2^{\circ} \mathrm{C}$, the mean effective temperature sum (the sum of daily mean temperatures $>5^{\circ} \mathrm{C}$ ) $750^{\circ} \mathrm{C}$ $\left(600-950^{\circ} \mathrm{C}\right)$ and the mean annual rainfall $450 \mathrm{~mm}$ in 1960-1990. The climatological data presented in Table 1 were calculated according to Ojansuu \& Henttonen (1983), and do not extend to Norway. More climatological data are provided in Kolkki (1966).

According to Kalela's (1961) Finnish forest site types the studied forests mainly represented UliginosumVaccinium-Empetrum type; a few forests were of Cladina type or Uliginosum-Empetrum-Myrtillus type, and two was of Ledum-Myrtillus type. Due to intensive reindeer grazing, the ground vegetation is very heterogeneous, however (Fig. 2). It is mainly composed of a mosaic CladinaCladonia-Dicranum-Pleurozium carpet. Further details concerning the vegetation are provided in Väre et al. (1995).

Scots pine is the dominant tree species at all sites and the only one at two of the sites. The age of the Scots pines varies between 100 and 200 years. Most of the sites also include Norway spruce (Picea abies, 5 sites) or downy birch (Betula pubescens, 9 sites), or both of these (3 sites) as an undergrowth (Table 1). In most cases Norway spruces and downy birches are seedlings or young saplings, under three metres in height. Hereafter, these tree species will be referred to as birch, pine and spruce.

Sporocarps of fungi were collected at 14 permanent sites (Fig. 1), once in 1991 and 1992 in the first week of September and twice in 1993 and 1994, between the last week of August and the second week of September. At site eight, sporocarps were collected in 1992-1994. Species occurring early or very late in the season may therefore not be represented in the data. The area of each study site was $600 \mathrm{~m}^{2}$. Sporocarps were dried in an airconditioned oven at $40^{\circ} \mathrm{C}$ for 1-2 days and weighed at room conditions. Biomasses are given in $\mathrm{g} \mathrm{dw} \mathrm{ha-1}$. Corticiaceous and most polyporous Aphyllophorales were collected only in 1994 and were not weighed. Life forms and mycorrhizal plant associates were evaluated on the basis of field notes and the literature (Trappe 1962, Molina et al. 1987, Hansen \& Knudsen 1992).

The total sporocarp yield of pine forests in the province of Lapland was estimated from information on the area of dry oligotrophic pine forest land and the age structure of stands for 1983-93 given in the Yearbook of Forest Statistics (Aarne 1994). Forests more than 40 years old were used in the calculations based on assumption that their fungal yield is similar to that of the aged stands of our study.

If a species occurred each year at each site, its occurrence percentage was 100 (see Table 2). A species was considered to be common (more precisely, widely distributed) at the study sites if it occurred at more than three sites. 


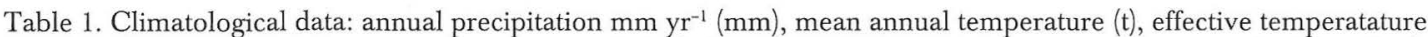
$\operatorname{sum}\left(\Sigma \mathrm{t}\right.$, the sum of daily average temperatures $>5^{\circ} \mathrm{C}$ ). Pine growth data: number of mature trees ha ${ }^{-1}$ (number), basal area $\mathrm{m}^{2} \mathrm{ha}^{-1}(\mathrm{ba})$, breast height diameter $\mathrm{cm}$ (bhd), height $\mathrm{m}(\mathrm{h})$, volume $\mathrm{m}^{3} \mathrm{ha}^{-1}$ (vol). Number of pine, birch and spruce saplings $\mathrm{ha}^{-1}$. Explanation of site numbers are given in Fig. 1.

\begin{tabular}{|c|c|c|c|c|c|c|c|c|c|c|c|}
\hline \multirow[t]{2}{*}{ Site } & \multicolumn{3}{|c|}{ climatological data } & \multicolumn{5}{|c|}{ pine growth data } & \multicolumn{3}{|c|}{ number of saplings } \\
\hline & $\mathrm{mm}$ & $\mathrm{t}$ & St & number & $\mathrm{ba}$ & bhd & $\mathrm{h}$ & vol & pine & birch & spruce \\
\hline 1 & 500 & -1.0 & 795 & 1231 & 13.7 & 11.9 & 10.6 & 80 & & 17 & \\
\hline 2 & 445 & -1.7 & 718 & 450 & 17.6 & 22.3 & 15.0 & 135 & 3333 & & \\
\hline 3 & 439 & -1.3 & 723 & 383 & 10.7 & 18.9 & 11.7 & 62 & 1333 & 17 & \\
\hline 4 & 393 & -1.7 & 660 & 633 & 11.8 & 15.4 & 8.7 & 60 & 333 & 67 & \\
\hline 5 & 393 & -1.8 & 628 & 500 & 13.7 & 18.7 & 8.3 & 64 & 83 & 100 & \\
\hline 6 & 418 & -1.5 & 644 & 467 & 8.5 & 15.2 & 7.2 & 33 & 833 & 67 & \\
\hline 7 & 517 & -1.4 & 751 & 133 & 6.3 & 24.5 & 14.4 & 43 & & & \\
\hline 8 & & & & 1667 & 17.9 & 11.7 & 8.4 & 82 & 50 & & \\
\hline 9 & 513 & 0.0 & 894 & 1017 & 16.5 & 14.4 & 11.0 & 94 & & 50 & \\
\hline 10 & 508 & -2.1 & 708 & 183 & 6.8 & 21.7 & 14.3 & 45 & 500 & 83 & 17 \\
\hline 11 & 513 & -0.9 & 781 & 600 & 23.2 & 22.2 & 15.2 & 180 & & 417 & 67 \\
\hline 12 & 511 & 0.8 & 985 & 667 & 22.2 & 20.6 & 12.1 & 137 & & & 67 \\
\hline 13 & 403 & -1.3 & 707 & 1017 & 11.7 & 12.1 & 8.4 & 55 & & 83 & \\
\hline 14 & 491 & -1.7 & 720 & 417 & 9.9 & 17.4 & 12.3 & 63 & & 100 & 33 \\
\hline
\end{tabular}

Fig. 2. A study site at Salla, Naruska, heavily grazed by reindeer (18.7.1990).

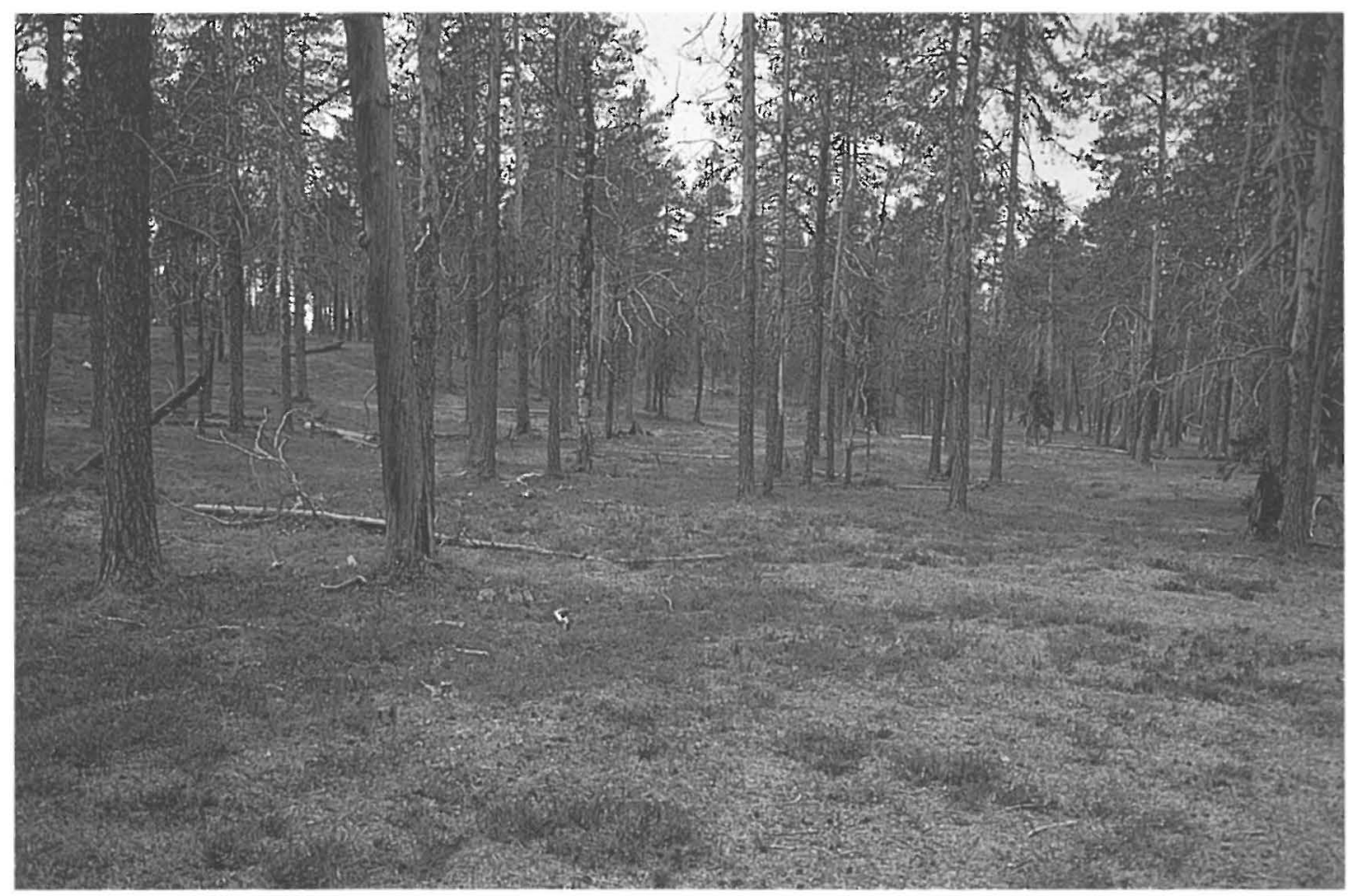


Microscopic measurements were made on samples embedded in Melzer solution, by using $100 ¥ 15$ magnification. Sporocarps from all stands in which the species was found were included in the measurements. Some Cortinarius of the subgenus Dermocybe were analysed by thin layer chromatography (Høiland 1984).

The nomenclature follows Hansen \& Knudsen (1995) for Ascomycota and Dacrymycetales, and Hansen \& Knudsen (1992) for Boletales, Agaricales and Russulales. Aphyllophorales sensu Jülich (1984) follows Gilbertson \& Ryvarden (1986) for families within polyporoid genera, Eriksson \& Ryvarden (1973, 1975, 1976), Eriksson et al. $(1978,1981,1984)$, Hjortstam et al. (1988) for species in Corticiaceae, and Hallenberg (1985) for species in Coniophoraceae. Nomenclature for polyporoid species was adopted from Niemelä (1993) and that for hydnaceous species from Niemelä \& Kotiranta (1993). Several collections are deposited in the herbarium, University of Oulu (OULU).

\section{Results and discussion}

A total of 207 taxa were found, 167 of them being stipitate and 40 resupinate or polyporous (Table 2). The maximum number of stipitate species (122) was found in 1993 (Table 3). Hintikka (1988) reported 80 stipitate species from pine forests in southern Finland, 75\% of the mycorrhizal species and 55\% of decomposers being the same as in the present study. Salo (1993) identified 232 macrofungi (Polyporales, Boletales, Agaricales, Russulales) from boreal upland forests and peatlands, including birch, pine and spruce forests. About $45 \%$ of these species were also found in the present study. Thus it seems that there is a fairly high overall similarity of the fungus species in different pine forest types in Finland. There was no clear geographical shift in the species composition from south to north; the number of species was lowest at Svanvik, Norway, which was the third northernmost study site.

Only five species belonged to Ascomycota, four of them to Helotiales, with two families and two genera. Cenococcum geophilum was a common imperfect species of Ascomycota. Basidiomycota included four classes: 1) Dacrymycetales with two species, 2) Aphyllophorales with seven families, 38 genera and 51 species, 3) Boletales with four families, seven genera and 13 species and 4) Agaricales with nine families, 34 genera and 135 species (Table 2). Two families, Cortinariaceae (52 species) and Tricholomataceae (44 species) included $47 \%$ of all species. Russulaceae in- cluded 18 identified species, Corticiaceae 17 and Poly-poraceae 16.

In total, 152 species were associated with pine. These included 90 mycorrhizal species, eight of them perhaps also mycorrhizal with birch or spruce, 44 wood decomposers and 18 pine litter decomposers (Table 4). These can be regarded as true specialists in northern oligotrophic pine forests. The seven common moss saprophytes (Table 4) may also occur in birch or spruce forests.

Although birch undergrowth was sparse, there were 26 species associated with it, 13 of them mycorrhizal and 13 wood decomposers (Table 4).

Four species were mycorrhizal with spruce and two wood decomposer were found growing on it. Living spruce, dead spruce trunks and other remnants of spruce were very rare at the study sites. In addition, there were 15 species which were mycorrhizal with birch, pine or spruce, or some combination of these (Table 4). In all 91 species were considered to be common at the study sites (Table 4); 72 species (including 22 resupinate or polyporous taxa) were found at only one site and 44 (including 10 corticious or polyporous taxa) were found at two or three sites (Table 2).

\section{Life forms}

Of all species, 115 (55\%) were mycorrhizal and 90 were decomposers (including 40 resupinate and polyporoid species). The ratio was thus 1.4 (Table 4). Sixty-two of the common species $(70 \%)$ were mycorrhizal and 28 were decomposers, for a ratio of 2.2. A higher proportion of the rare species were decomposers, taking advantage perhaps of rare substrates. The relationships is opposite to that of mesic sites, where decomposer species are more abundant (Ohenoja 1993, Ohenoja \& Väre 1993, Salo 1993). This is mainly due to the more diverse substrate selection possibilities and the higher humidity than at the present study sites. Mossy sites (almost continuous Pleurozium schreberi carpet) always included more diverse decomposer fungal flora than did lichen dominated sites.

Mycorrhizal species. Ninety mycorrhizal species (including eight uncertain) were associated with 
Table 2. Fungal species, and in parentheses number of species in taxonomic group. Yield $\left(g \mathrm{dw}^{\mathrm{h}} \mathrm{a}^{-1}\right)$ is given as mean weight, maximum site specific weight ( $\max$ ) and maximum annual mean weight ( $\max$ annu) for a particular year (yr) of measurement. The sum of the yields is given separately for species-rich genera and family. Occurrence is given as $\%$ of collections (4 years $¥ 14$ sites), as maximum number of years (no yr) at a specific site and number of sites (no) at which species seen. Life forms are B, P, S = mycorrhizal with birch, pine and spruce, respectively; $\mathrm{Pn}=$ saprophytic in pine needles; $\mathrm{M}=$ saprophytic in mosses; $\mathrm{Bw}, \mathrm{Pw}$ and $\mathrm{Sw}=$ saprophytic on birch, pine and spruce wood, respectively. $\mathrm{Bl}=$ basidiolichen, $\mathrm{Co}=$ coprophilous, $\mathrm{My}=$ mycophilous.

\begin{tabular}{|c|c|c|c|c|c|c|c|c|}
\hline \multirow[t]{2}{*}{ Order/Class/Family/Species } & \multicolumn{3}{|c|}{ yield } & & \multicolumn{3}{|c|}{ occurrence } & life form \\
\hline & $\begin{array}{c}\text { mean } \\
\text { weight }\end{array}$ & $\begin{array}{r}\max \\
\text { site }\end{array}$ & $\begin{array}{l}\max \\
\text { annu }\end{array}$ & $\mathrm{yr}$ & $\%$ & $\begin{array}{l}\text { no } \\
\text { yr }\end{array}$ & no & \\
\hline
\end{tabular}

\section{ASCOMYCOTA (5)}

Helotiales (4)

Geoglossaceae (2)

Spathularia flavida Pers.:Fr.

S. rufa Schmidel

Leotiaceae (2)

Cudonia circinans (Pers.:Fr.) Fr.

C. confusa Bres.

Fungi Imperfecti (1)

Cenococcum geophilum Fr.

BASIDIOMYCOTA (202)

Dacrymycetales (2)

Dacrymycetaceae (2)

Dacrymyces stillatus Nees:Fr.

D. tortus (Willd.:Fr.) Fr.

Aphyllophorales (50)

Clavariaceae (2)

Clavariadelphus sp.

Clavulinopsis sp.

Clavulinaceae (1)

Clavulina cristata (Fr.) Schröt.

Thelephoraceae (7)

Bankera fuligineoalba (Schmidt.:Fr.) Pouzar

Boletopsis grisea (Peck) Bondartsev \& Singer

Hydnellum caeruleum (Hornem.) P. Karst.

H. ferrugineum (Fr.:Fr.) P. Karst.

H. peckii Banker ex Peck

Phellodon tomentosus (L.:Fr.) Banker

Thelephora terrestris Pers. ex Fr. 1
1.48

0.12

0.12

0.006

$\begin{array}{lll}7 & 0.5 & 93\end{array}$

$\begin{array}{lll}3.5 & 0.2 \quad 93\end{array}$

1.8

1.8

1

1

B, P, S

1.36

0.26

1.1

$\begin{array}{ll}0.06 \quad 93 \\ 3 & 93\end{array}$

\section{6}

12.5

11

B, P, S

B, P, S

B, P, S - 
Table 2. Contd.

Hyphodontia aspera (Fr.) J. Erikss.

H. subalutacea (P. Karst.) J. Erikss.

Phanerochaete sanguinea (Fr.) Pouz.

Piloderma croceum J. Erikss. \& Hjortstam

Resinicium furfuraceum (Bres.) Parmasto

Sistotremastrum suecicum Litsch. ex J. Erikss.

Sphaerobasidium minutum (J. Erikss.) Oberw. ex Jülich

Stereum hirsutum (Willd.:Fr.) Gray

Trechispora farinacea (Pers.:Fr.) Liberta s. str.

T. subsphaerospora (Litsch.) Liberta

Coniophoraceae (3)

Coniophora arida (Fr.) P. Karst.

C. fusispora (Cooke \& Ellis) Sacc.

Leucogyrophana pulverulenta (Fr.) Ginns

Pw

$\mathrm{Pw}$

$\mathrm{Pw}$

Pw

$\mathrm{Pw}$

$\mathrm{PW}_{\mathrm{W}}$

$\mathrm{Pw}$

$\mathrm{Bw}$

$\mathrm{Pw}_{w}$

$\mathrm{PW}$

Pw

Pw

$\mathrm{Pw}$

Hymenochaetaceae (3)

Coltricia perennis (L.:Fr.) Murrill

44

44

286

53

93

62.5

$\mathrm{P}, \mathrm{Pn}$

$\mathrm{Bw}$

$\mathrm{Pw}$

$P$. viticola (Schwein. ex Fr.) Donk

Polyporaceae (16)

Amyloporia xantha (Fr.:Fr.) Bondartsev \& Singer

Antrodia albobrunnea (Romell) Ryvarden

Fomes fomentarius (L.:Fr.) Fr.

Fomitopsis pinicola (Sw.:Fr.) P. Karst.

Gloeophyllum sepiarium (Wulfen:Fr.) P. Karst.

Junghuhnia luteoalba (P. Karst.) Ryvarden

Oligoporus sericeomollis (Romell) Bondartseva

Piptoporus betulinus (Bull.:Fr.) P. Karst.

Polyporus brumalis (Pers.:Fr.) Fr.

25

0.7

91

1.8

Pw

Pw

Bw

Pw

Pw

Pw

Pw

$\mathrm{Bw}$

Bw

Pw

Pw

$\mathrm{Sw}$

Pw

Pw

Sw

PW

T. hollii (J. C. Schmidt:Fr.) Kreisel

Boletales (13)

1375

Paxillaceae (1)

Paxillus involutus (Batsch:Fr.) Fr.

8

310

30

92

10.7

$\mathrm{P}$

Boletaceae (5)

Boletus subtomentosus L.: Fr. coll.

68

Leccinum spp.

Leccinum scabrum (Bull.:Fr.) Gray coll.

2.3

65

L. variicolor Watling

L. versipelle (Fr.) Snell

L. vulpinum Watling

17

0.1

31

17

70

5

91

$$
5.4
$$

$340 \quad 36$

$\begin{array}{ll}5 & 0.3\end{array}$

$360 \quad 52$

570

69

93

92

93

93

10.7

1.8

17.9

10.7

0.8

Rhizopogonaceae (1)

Rhizopogon vulgaris (Vitt.) M. Lange

0.8

45

45

3

92

1.8

$\mathrm{B}, \mathrm{P}$

Chroogomphus rutilus (Schaeff.:Fr.) O. K. Miller 33.7

Gomphidius glutinosus (Schaeff.:Fr.) Fr.

G. roseus (Fr.) Fr.

Suillus spp.

Suillus bovinus (L.:Fr.) Roussel 
Table 2. Contd.

S. luteus (L.:Fr.) Roussel

S. variegatus (Sw.:Fr.) O. Kuntze

Agaricales (137)

Hygrophoraceae (7)

Hygrocybe miniata (Fr.) P. Kumm.

H. psittacina (Schaeff.:Fr.) P. Kumm.

Hygrophorus spp.

Hygrophorus agathosmus (Fr.) Fr.

H. camarophyllus (Alb. \& Schwein.:Fr.)

Dumèe et al.

H. hypothejus (Fr.:Fr.) Fr.

H. karstenii Sacc. \& Cub.

H. olivaceoalbus (Fr.:Fr.) Fr.

Tricholomataceae (44)

Armillaria borealis Marxmüller \& Korhonen

Cantharellula umbonata (Gmel.:Fr.) Singer

Clitocybe diatreta (Fr.:Fr.) Gillet

C. vibecina (Fr.) Quél.

Collybia spp.

Collybia butyracea (Bull.:Fr.) P. Kumm.

C. distorta (Fr.) Quél.

C. maculata (Alb. \& Schw.:Fr.) P. Kumm.

C. peronata (Bolt.:Fr.) P. Kumm.

C. putilla (Fr.:Fr.) Singer

C. racemosa (Pers.:Fr.) Quél.

C. succinea (Fr.) Quél.

C. tuberosa (Bull.:Fr.) P. Kumm.

Cystoderma granulosum (Batsch:Fr.) Kühner $<0.00$

C. jasonis (Cooke \& Massee) Harmaja

Laccaria bicolor (Maire) Orton

Lyophyllum spp.

Lyophyllum decastes (Pers.:Fr.) Orton

L. fumosum (Fr.:Fr.) Singer

L. semitale (Fr.) Kühner

Marasmius androsaceus (L.:Fr.) Fr.

Mycena spp.

Mycena clavicularis (Fr.) Gillet

M. epipterygia (Scop.:Fr.) Gray

M. galericulata (Scop.:Fr.) Gray

$M$. galopus (Pers.:Fr.) P. Kumm.

M. haematopus (Pers.:Fr.) P. Kumm.

$M$. laevigata (Lasch:Fr.) Gillet

M. sanguinolenta (Alb. \& Schwein.:Fr.)

P.Kumm.

M. viridimarginata $\mathrm{P}$. Karst.

Omphaliaster asterosporus (J. E. Lange)

Lamoure

Omphalina umbellifera (L.:Fr.) Quél.

Ripartites sp.

Tricholoma spp.

Tricholoma aestuans (Fr.) Gillet

T. auratum (Fr.) Gillet ss. Bon

T. focale (Fr.) Ricken

T. imbricatum (Fr.:Fr.) P. Kumm.

T. nauseosum (Blytt) Kytövuori

T. pessundatum (Fr.) Quél.

T. portentosum (Fr.) Quél.

T. saponaceum (Fr.:Fr.) P. Kumm.

$<0.00$

0.9

$$
\begin{array}{r}
2.6 \\
1200 \\
3090
\end{array}
$$

1.5

0.2

0.8

0.12
0.3

0.12

$$
2.5
$$

0.3

0.2
0.02

93

1.8

12.5

62.5

$\mathrm{P}$

$\mathrm{P}$

200

$2.0 \quad 110$

$\begin{array}{ll}3.5 & 76\end{array}$

0.7

6.4

14

0.6

0.7

5.1

0.12

4.7

0.001

0.6

1.8

$<0.001$
3.2

3.2
25

58

$20 \quad 575$

$38 \quad 455$

$2.3 \quad 75$

$1.1 \quad 15$

2.8

1.3
0.003

0.003
0.004

$<0.001$

0.11

25

0.17

1.5

0.17

0.4

93

1.8

M

M

3.6

S

1.8

1.8

1.8

B, S

(P), $\mathrm{P}$

$\begin{array}{rrr}1 & 91 & 1.8 \\ 93 & 0.5 & 1.8\end{array}$

B

$1.3 \quad 25$

1.8

14.3

14.3

44.6

14.3

3.6

18

3.6

0.3

16

0.06

9
1.8

12.5

42.8

1.8

39.3

67.9

7.1

19.6

12.5

21.4

35.7

0.01

0.1

0.02

0.04

$<0.001$

$<0.001$

0.3
0.17

0.02

0.01

0.001

0.3

77

$8.8 \quad 330$

0.06

0.8
15

2

93
93

93

35.7
1.8

5.4

3.6

3.6

12.5

1.8

1.8

1.8

32.1

1.8

5.6

17.9

7.1

1.8

1.8

5.6

1.8

5.6
B
M
M
Pn

Pn
Pn
Pn
M
Pn
M
Pn
My
M
M
P

$\mathrm{P}$
$\mathrm{P}$

Pn

Pn

Pn

Pn

$\mathrm{Bw}$

Pn

Bw

Pw

Pw

Pw

M
Bl

Pn

$\mathrm{P}$
$\mathrm{P}$
$\mathrm{P}$
$\mathrm{P}$
$\mathrm{P}$
$\mathrm{P}$
$\mathrm{P}$
$\mathrm{P}$ 
Table 2. Contd.

T. stans (Fr.) Sacc.

$T$. virgatum (Fr.:Fr.) P. Kumm.

T. viridilutescens Moser

Tricholomopsis decora (Fr.) Singer

T. rutilans (Schaeff.:Fr.) Singer

Xeromphalia campanella (Batsch:Fr.)

Kühner \& Maire

Pluteaceae (1)

Pluteus atricapillus (Batsch) Fayod

Coprinaceae (1)

Psathyrella sp.

Strophariaceae (9)

Flammulaster limulatoides Orton

Hypholoma capnoides (Fr.) P. Kumm.

Phaeomarasmius borealis Rald

Pholiota spp.

Pholiota alnicola (Fr.:Fr.) Singer

$P$. heteroclita (Fr.:Fr.) Quél.

P. lubrica (Pers.:Fr.) Singer

P. mixta (Fr.) Singer

Stropharia hornemannii (Fr.:Fr.) Lundell

S. semiglobata (Batsch:Fr.) Quél.

Cortinariaceae (52)

Cortinarius spp.

Cortinarius adalberti Favre

C. armeniacus Fr.

C. armillatus (Fr.:Fr.) Fr.

C. balteatus (Fr.) Fr.

C. biformis Fr.

C. brunneus (Pers.:Fr.) Fr.

C. camphoratus (Fr.) Fr.

C. cinnamomeus (L.:Fr.) Fr.

C. claricolor (Fr.) Fr.

C. collinitus (So:v.:Fr.) Fr. ss. J. E. Lange

C. colus Fr.

C. croceus (Schaeff.) Bigeard \& Guill.

C. gentilis (Fr.) Fr.

C. huronensis Ammirati \& A. M. Sm. var. olivaceus Ammirati \& A. M. Sm.

C. cf. incisus (Pers.:Fr.) Fr.

C. cf. laetus Moser

C. leucophanes P. Karst.

C. lux-nymphae Melot

C. mucosus (Bull.:Fr.) Kickx

C. niveoglobosus Lindstr.

C. obtusus (Fr.) Fr.

C. ochrophyllus Fr.

C. odhinnii Melot

C. paleaceus Fr.

C. paragaudis Fr. subsp. paragaudis

C. pholideus (Fr.:Fr.) Fr.

C. querciticus $\mathrm{H}$. Lindstr.

C. raphanoides (Pers.:Fr.) Fr.

C. scaurus (Fr.:Fr.) Fr.

C. semisanguineus (Fr.) Gillet

C. stillatitius $\mathrm{Fr}$.

C. talus Fr.

$\begin{array}{rrrrrrrr}5.1 & 150 & 18 & 92 & 3.6 & 1 & 2 & \mathrm{P} \\ 1.8 & 100 & 7 & 93 & 1.8 & 1 & 1 & \mathrm{P} \\ 11 & 475 & 34 & 92 & 3.6 & 2 & 1 & \mathrm{P} \\ 0.2 & 6.5 & 0.55 & 92 & 3.6 & 1 & 2 & \mathrm{Pw} \\ 3.2 & 7.5 & 11 & 93 & 5.4 & 2 & 2 & \mathrm{Pw} \\ 1.8 & 35 & 5 & 93 & 19.6 & 2 & 7 & \mathrm{Pw} \\ & & & & & & & \\ 0.8 & & & & & & & \\ 0.8 & 30 & 2 & 94 & 10.7 & 1 & 2 & \mathrm{Bw} \\ & & & & & & & \\ 0.002 & & & & & & & \\ 0.002 & 1.2 & 0.08 & 92 & 1.8 & 1 & 1 & \mathrm{Pn} \\ & & & & & & & \\ 23 & & & & & & & \mathrm{Pw} \\ 0.03 & 25 & 1 & 91 & 3.6 & 1 & 2 & \mathrm{Pw} \\ 23 & 325 & 3 & 93 & 23.2 & 4 & 8 & \mathrm{Bw} \\ <0.001 & 0.17 & 0.01 & 94 & 1.8 & 1 & 1 & \mathrm{Bw} \\ 27 & & & & & & & \mathrm{Bw} \\ 18 & 395 & 28 & 94 & 14.3 & 3 & 6 & \mathrm{Bw} \\ 0.8 & 45 & 3 & 91 & 1.8 & 1 & 1 & \mathrm{M} \\ 1.2 & 50 & 5 & 91 & 3.6 & 1 & 2 & \\ 6.8 & 110 & 19 & 93 & 37.5 & 4 & 11 & 1 \\ 1.8 & 75 & 5 & 91 & 3.6 & 2 & 1 & 1 \\ 0.001 & 1 & 0.06 & 91 & 1.8 & 1 & 1 & \end{array}$

985

940

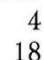

18

43

12

93

170

12

8.1

17

6.6

0.4

22

20

50

270

766

154

755

1010

310

67

300

165

6.5

150

300

$$
10
$$

30

92

31

67

327

24

19

46

14

0.8

35

30

0.15

0.9

0.003

7.5

30

30

0.1

425

433

460

$\begin{array}{rr}68 & 460\end{array}$

$16 \quad 185$

$17 \quad 310$

13160

$0.8 \quad 36$

$15 \quad 135$

$5.5 \quad 60$

$24 \quad 410$

$27 \quad 730$

$51 \quad 435$

$140 \quad 990$

3

0.1

61
72

112

112
2

33

30

37

3

29

6
42

42
82

98

243

3.3

5.5

\section{6}

25.0

30.3

16.1

220

71.4

10.7

46.4

19.6

12.5

21.4

73.2

37.5

5.4

10.7

1.8

50

44.6

60.7

1.8

60.7

16.1

42.9

7.1

25.0

17.9

12.5

16.1

62.5

76.8

16.1

5.4
$P$
$P$
$P$
$P w$
$P w$
$P w$

$B w$

$P n$

$P w$
$P w$
$B w$
$B w$
$B w$
$B w$
$P n$
$M$
$C o$$$
\text { Co }
$$$$
\begin{array}{r}
50 \\
200
\end{array}
$$$$
\begin{array}{r}
\mathrm{P} \\
\mathrm{P} \\
\mathrm{B} \\
\mathrm{P} \\
\mathrm{P} \\
\mathrm{P} \\
\mathrm{P} \\
\mathrm{P} \\
\mathrm{P} \\
\mathrm{P} \\
\mathrm{P} \\
\mathrm{P} \\
\mathrm{P}, \mathrm{S}
\end{array}
$$

$\begin{array}{rrr}1 & 2 & \mathrm{P} \\ 2 & 9 & \mathrm{P} \\ 4 & 7 & \mathrm{~B} \\ 3 & 6 & \mathrm{P} \\ 9 & 14 & \mathrm{P} \\ 4 & 14 & \mathrm{P} \\ 3 & 4 & \mathrm{P} \\ 3 & 13 & \mathrm{P} \\ 3 & 6 & \mathrm{P} \\ 2 & 5 & \mathrm{P} \\ 2 & 6 & \mathrm{P} \\ 4 & 14 & \mathrm{P} \\ 2 & 12 & \mathrm{P}, \mathrm{S} \\ & & \mathrm{P} \\ 1 & 3 & \mathrm{P} \\ 1 & 6 & \mathrm{P} \\ 1 & 1 & \mathrm{P} \\ 3 & 13 & \mathrm{P} \\ 4 & 12 & \mathrm{P} \\ 4 & 14 & \mathrm{P} \\ 2 & 1 & \mathrm{P} \\ 3 & 14 & \mathrm{P} \\ 2 & 5 & \mathrm{P} \\ 4 & 12 & \mathrm{P}) \\ 2 & 3 & \mathrm{P} \\ 4 & 10 & \mathrm{P} \\ 3 & 6 & \mathrm{P} \\ 4 & 4 & \mathrm{P} \\ 3 & 6 & \mathrm{P}) \\ 4 & 14 & \\ 4 & 14 & 5\end{array}$


Table 2. Contd.

C. traganus (Fr.:Fr.) Fr.

C. vibratilis (Fr.) Fr.

Cortinarius sp.

Galerina spp

Galerina atkinsoniana A. M. Sm

G. calyptrata Orton

G. camerina (Fr.) Kühner

G. marginata (Batsch) Kühner

G. mniophila (Lasch) Kühner

G. pumila (Pers.:Fr.) Singer

Gymnopilus penetrans (Fr.) Murr.

Hebeloma spp.

Hebeloma birrus (Fr.) Sacc.

H. bryogenes Vesterholt

$H$. leucosarx Orton

Hebeloma sp.

Inocybe spp.

Inocybe jacobi Kühner

I. lacera (Fr.) P. Kumm.

I. leptophylla G. F. Atk.

I. mixtilioides Kuyper ined.

I. soluta Velen.

I. subcarpta Kühner \& Boursier

Rozites caperatus (Pers.:Fr.) Fr.

Crepidotaceae (1)

Tubaria confragosa (Fr.) Kühner

Entolomataceae (2)

Entoloma cetratum (Fr.:Fr.) Moser E. turbidum (Fr.) Quél.

Russulales (18)

Russulaceae (18)

Lactarius spp.

Lactarius helvus (Fr.) Fr.

L. mammosus (Fr. ex Weinm.) Fr.

L. musteus Fr.

L. necator (J. F. Gmel.:Fr.) Pers.

L. rufus (Scop.:Fr.) Fr.

L. trivialis (Fr.:Fr.) Fr.

L. vietus (Fr.) Fr.

Russula spp.

Russula adusta Fr.

$R$. aquosa Leclair

$R$. claroflava Grove

$R$. consobrina (Fr.:Fr.) Fr.

$R$. decolorans (Fr.) Fr.

R. paludosa Britz.

$R$. roseipes Bres.

R. turci Bres. ss. Maire

$R$. vinosa Lindbl.

R. xerampelina (Schaeff.) Fr. coll.

$R$. sp. sectio Russula

\begin{tabular}{|c|c|c|c|c|c|c|c|}
\hline 52 & 75 & 104 & 93 & 33.9 & 3 & 11 & $\mathrm{P}$ \\
\hline 17 & 260 & 52 & 92 & 53.4 & 3 & 14 & $P$ \\
\hline 2.6 & 100 & 12 & 92 & 3.6 & 1 & 2 & $\mathrm{P}$ \\
\hline \multicolumn{8}{|l|}{2.2} \\
\hline 1.3 & 20 & 3 & 93 & 44.6 & 4 & 14 & M \\
\hline 0.17 & 3 & 0.5 & 93 & 17.9 & 4 & 8 & M \\
\hline 0.002 & 1.3 & 0.1 & 91 & 3.6 & 1 & 1 & Pw \\
\hline 0.02 & 6.5 & 0.05 & 91 & 3.6 & 1 & 2 & $\mathrm{Pw}$ \\
\hline 0.42 & 5.5 & 0.6 & 92 & 33.9 & 4 & 12 & M \\
\hline 0.19 & 5.5 & 61 & 93 & 19.6 & 3 & 8 & M \\
\hline 11 & 230 & 31 & 93 & 48.2 & 3 & 14 & Pn \\
\hline \multicolumn{8}{|l|}{11} \\
\hline 0.003 & 2 & 0.1 & 93 & 1.8 & 1 & 1 & $\mathrm{P}$ \\
\hline 2.1 & 65 & 4 & 93 & 5.4 & 1 & 3 & $\mathrm{P},(\mathrm{B})$ \\
\hline 8.7 & 200 & 22 & 92 & 12.5 & 3 & 5 & $\mathrm{P},(\mathrm{B})$ \\
\hline 0.003 & 1.5 & 1.5 & 92 & 1.8 & 1 & 1 & $\mathrm{P}$ \\
\hline \multicolumn{8}{|l|}{11} \\
\hline 0.003 & 0.15 & 0.01 & 93 & 1.8 & 1 & 1 & $\mathrm{P}$ \\
\hline 0.005 & 1.5 & 0.02 & 93 & 5.4 & 1 & 3 & $\mathrm{P}$ \\
\hline 1.5 & 75 & 5.5 & 93 & 5.4 & 1 & 2 & $\mathrm{P}$ \\
\hline 0.005 & 2.5 & 0.2 & 94 & 1.8 & 1 & 1 & $\mathrm{P}$ \\
\hline 7.5 & 70 & 10 & 94 & 33.9 & 4 & 9 & $\mathrm{P}$ \\
\hline 1.6 & 25 & 3 & 92 & 12.5 & 3 & 4 & $\mathrm{P}$ \\
\hline 9.2 & 110 & 12 & 93 & 12.5 & 2 & 4 & B \\
\hline \multicolumn{8}{|l|}{0.2} \\
\hline 0.2 & 10 & 0.7 & 93 & 1.8 & 1 & 1 & $\mathrm{Bw}$ \\
\hline \multicolumn{8}{|l|}{1.5} \\
\hline 1.4 & 25 & 3 & 92 & 44.6 & 4 & 10 & $\mathrm{P}$ \\
\hline 0.06 & 25 & 0.15 & 91 & 5.4 & 1 & 3 & (B) \\
\hline
\end{tabular}

1880

1200

\begin{tabular}{rrrrrrrr}
1.5 & 45 & 6 & 91 & 3.6 & 1 & 2 & $\mathrm{P}$ \\
24 & 150 & 38 & 93 & 48.2 & 1 & 11 & $\mathrm{P}$ \\
4.1 & 70 & 9 & 93 & 8.9 & 2 & 4 & $\mathrm{P}$ \\
2.2 & 125 & 9 & 91 & 1.8 & 1 & 1 & $\mathrm{~B}$ \\
1100 & 8630 & 2495 & 93 & 87.5 & 2 & 13 & $\mathrm{P}$ \\
0.3 & 18 & 1 & 91 & 1.8 & 1 & 1 & $\mathrm{~B}$ \\
4.4 & 110 & 0.5 & 93 & 10.7 & 4 & 3 & $\mathrm{~B}$ \\
680 & & & & & & & \\
3.0 & 165 & 12 & 91 & 1.8 & 1 & 1 & $\mathrm{P}$ \\
1.0 & 30 & 2 & 93 & 3.6 & 2 & 1 & $\mathrm{~S}$ \\
12 & 125 & 28 & 93 & 16.1 & 3 & 6 & $\mathrm{~B}$ \\
5.2 & 135 & 18 & 93 & 5.4 & 1 & 3 & $\mathrm{P}$ \\
340 & 3400 & 1095 & 93 & 39.3 & 3 & 12 & $\mathrm{P}$ \\
250 & 3450 & 785 & 93 & 23.2 & 4 & 9 & $\mathrm{P}$ \\
0.2 & 10 & 0.7 & 92 & 1.8 & 1 & 1 & $\mathrm{~S}$ \\
1.2 & 25 & 5 & 93 & 5.4 & 1 & 3 & $\mathrm{P},(\mathrm{S})$ \\
73 & 1235 & 170 & 93 & 19.6 & 3 & 7 & $\mathrm{P}$ \\
0.3 & 17 & 1.2 & 92 & 1.8 & 1 & 1 & $\mathrm{~B}, \mathrm{P}, \mathrm{S}$ \\
1.9 & 40 & 5 & 94 & 7.1 & 1 & 4 & $\mathrm{~B},(\mathrm{P})$ \\
\hline
\end{tabular}


Table 3. Annual yield ( $\mathrm{g} \mathrm{dw} \mathrm{ha}^{-1}$ ) and number (no) of species of decomposer (D) and mycorrhizal (M) fungi in 19911994. Maximum yield (max yield) indicates number of species that had their highest yield in the given year. Common species include edible and mycorrhizal Lactarius rufus, Russula decolorans, R. paludosa and Suillus variegatus.

\begin{tabular}{|c|c|c|c|c|c|c|c|c|c|c|}
\hline \multirow{3}{*}{ Year } & \multirow{2}{*}{\multicolumn{2}{|c|}{ yield }} & \multirow{2}{*}{\multicolumn{2}{|c|}{ no }} & \multirow{2}{*}{\multicolumn{2}{|c|}{ total }} & \multirow{2}{*}{\multicolumn{2}{|c|}{$\begin{array}{l}\max \\
\text { yield }\end{array}$}} & \multicolumn{2}{|c|}{ common species } \\
\hline & & & & & & & & & \multirow{2}{*}{$\begin{array}{c}\text { yield } \\
\mathrm{g} \mathrm{ha}^{-1}\end{array}$} & \multirow{2}{*}{$\frac{\text { proportion }}{\%}$} \\
\hline & $\mathrm{D}$ & $\mathrm{M}$ & $\mathrm{D}$ & $\mathrm{M}$ & yield & no & $\mathrm{D}$ & $\mathrm{M}$ & & \\
\hline 1991 & 105 & 2940 & 31 & 80 & 3045 & 111 & 12 & 20 & 1430 & 47 \\
\hline 1992 & 85 & 4445 & 31 & 85 & 4530 & 116 & 15 & 39 & 1970 & 44 \\
\hline 1993 & 155 & 9530 & 32 & 90 & 9685 & 122 & 18 & 54 & 7560 & 77 \\
\hline 1994 & 70 & 52 & 24 & 52 & 1115 & 76 & 6 & 4 & 615 & 55 \\
\hline Mean & 104 & 4490 & 30 & 77 & 4594 & 107 & & & 2870 & 62 \\
\hline
\end{tabular}

Table 4. Number of species of different life forms. The number of common species is given in parentheses*. BL $=$ basidiolichen, Dtot $=$ total number of decomposers, $\mathrm{Dw}=$ wood decomposers, $\mathrm{Dn}=$ needle decomposers, $\mathrm{Dm}=$ moss decomposers, $\mathrm{M}=$ mycorrhizal species. Miscellaneous (Misc) mycorrhizal species are those for which the tree associate remained unknown, and miscellaneous decomposers include one coprophilous and one mycophilous species, and 14 moss decomposers. Two species occurred in two categories, making the sum of species 209 and 91 , not 207 and 89 .

\begin{tabular}{|c|c|c|c|c|c|c|c|c|c|c|c|c|c|}
\hline \multirow{2}{*}{$\frac{\text { Associate }}{\text { Pine }}$} & \multirow[t]{2}{*}{$\mathrm{BL}$} & \multicolumn{2}{|c|}{ Dtot } & \multicolumn{2}{|c|}{ Dw } & \multicolumn{2}{|c|}{ Dn } & \multicolumn{2}{|c|}{$\mathrm{Dm}$} & \multicolumn{2}{|c|}{ M } & \multicolumn{2}{|c|}{ Total } \\
\hline & & 63 & $(20)$ & 45 & (9) & 18 & (11) & & & 83 & $(52)$ & 146 & (72) \\
\hline Birch & & 13 & (1) & 13 & (1) & & & & & 13 & (18) & 26 & (9) \\
\hline Spruce & & 1 & & 1 & & & & & & 4 & & 5 & \\
\hline Misc & $1(1)$ & 16 & $(7)$ & & & & & 14 & $(7)$ & 15 & $(2)$ & 32 & $(10)$ \\
\hline Total & $1(1)$ & 93 & (28) & 59 & (10) & 18 & (11) & 14 & (7) & 115 & $(62)$ & 209 & (91) \\
\hline
\end{tabular}

* Species that occurred at over three sites were regarded as common.

pine, 13 with birch, four with spruce and 15 with some combination of these (Table 4). About 10 mycorrhizal species within the mycorrhizal genera Cortinarius, Entoloma, and Hebeloma remained unidentified. Some 130 additional mycorrhizal fungal species amongst Agaricales and Boletales are associated with pine in Finland (Hansen \& Knudsen 1992). These occur mainly on calcareous or mesic soil, or are mainly restricted to southern Finland. If mycorrhizal species of Ascomycota and Aphyllophorales are added as well, there are 250-300 mycorrhizal species associated with pine in Finland.

Hypogeous Cenococcum geophilum (Ascomycota, Fungi Imperfecti) and Piloderma croceum (Corticiaceae) occurred at all sites. These mycorrhizal species could be identified on the surface of pine root tips on the basis on rigid deeply intensively coloured black and yellow hyphae, respectively. At five sites $P$. croceum also produced sporocarps on a lower surface of decayed decorticated pine. $C$. geophilum has been found only in sterile form on the root surface of various tree species and as sclerotia in soil (Trappe 1971). Sclerotia were found in abundance in the present study.

All Ascomycota (1 common), Boletales (8 common) and Russulales (8 common) were mycorrhizal. In Boletales the overwhelmingly most common species was Suillus variegatus, and in Russulales Lactarius rufus, Russula decolorans and R. paludosa (Table 2). Aphyllophorales included both mycorrhizal $(6 \mathrm{com}-$ mon) and decomposer species (10 common, all corticioid or polyporoid). The concept of life forms within Corticiaceae s. lat. is developing, however. The following genera have been reported to include mycorrhizal species: Am-phinema, Byssocorticium, Ceratobasidium, Pilo- 
derma, Sistotrema, Thanateporus, Tylospora and Ypsilonidium (Taylor \& Alexander 1991, Currah \& Zelmer 1992). All these produce typical resupinate sporocarps on various plant material. All Thelephoraceae found in the present study were mycorrhizal with pine. Although the mycorrhizal status of Thelephoraceae has sometimes been questioned (Maas Geesteranus 1975), there now seems to be a consensus on the matter. The mycorrhiza of Thelephoraceae has been described in several instances (Agerer 1993) as well as its function (Entry et al. 1991).

Thelephora terrestris produced sporocarps at only one site. We were surprised to find the sporocarps so rare since these are common in manured soil in northern Finland, often in clear cut areas that have been ploughed (Väre 1989). Most of the sites in our study are heavily grazed by reindeer, and breaking of the soil surface could be suspected to stimulate sporocarp formation. Coltricia perennis is one of the characteristic species in lichen-rich pine forests of northern Finland. It is both mycorrhizal and saprophytic (Danielson 1984), a very peculiar feature, especially for a polyporoid species.

Among the Agaricales, Cortinariaceae and Tricholomataceae included both mycorrhizal and saprophytic species (35 common). Mycorrhizal species were especially common in the genus Cortinarius (29 common species, $45 \%$ of all common mycorrhizal species) and it may be described as a characteristic genus in northern pine forests in northeastern Fennoscandia, although fungal yield is higher in the genera Suillus, Lactarius and Russula. In Tricholomataceae there were four common mycorrhizal species, e.g. Laccaria bicolor. The number of Tricholoma species was 11, but only T. auratum was common. Hygrophoraceae was rare in dry pine forests. Entoloma cetratum in Entolomataceae is considered to be mycorrhizal, although the life form of most Entoloma is unknown.

Decomposer species. Humicular saprophytic species (all Agaricales) may be divided into two categories, 18 of them decomposing pine needles and 14 of them mosses (Table 4). The following families were represented: Cortinariaceae with four common moss decomposers, all Galerina, Tricholomataceae with 20 species
(11 common species) and Strophariaceae with three species (one common, Pholiota mixta) (Table 2). Tricholomataceae included $75 \%$ of the humicular saprophytic species.

Cantharellula umbonata was the most productive moss decomposer. Ohenoja (1995) proposes that this species is mycorrhizal, but this should be studied. C. umbonata is fairly common (not every year) in Finland, but was infrequent in the present study. C. umbonata often grows amongst Dicranum, Pleurozium and Polytrichum. These mosses were reduced in size at most sites, however, due to trampling by reindeer, and $C$. umbonata was more common only at sites where grazing by reindeer was less intensive. Clitocybe diatreta and Cystoderma jasonis were other common moss decomposers.

Wood decomposers consisted of 58 (73\% of all decomposers) species in Dacrymycetales, Aphyllophorales and Agaricales (Table 2). Forty-six species were growing on pine (Table 4). These included 37 Aphyllophorales, 12 of which were common species in Corticiaceae and Polyporaceae. The most common species were Amyloporia xantha, Botryobasidium botryosum, Chaetoderma luna, Hyphoderma praetermissum, Phanerochaete sanguinea, Resinicium furfuraceum and Sistotremastrum suecicum.

There were only few fallen trunks at the study sites. The average number of resupinate decomposer species per site was 6.5. Many species in this group are considered to be common in old dry pine heaths (Eriksson \& Ryvarden 1973, 1975, 1976, Eriksson et al. 1978, 1981, 1984, Kotiranta \& Niemelä 1981, Hallenberg 1985, Hjortstam et al. 1988, Niemelä 1993, Renvall et al. 1991, Renvall 1995). Among the rarer species, Coniophora fusispora, Phellinus viticola and Skeletocutis stellae were found at Raja-Jooseppi, Boletopsis grisea (ectomycorrhizal), Skeletocutis lenis, and S. subincarnata at Aska, S. lilacina at Lokka, Postia cf. lateritia at Muonio and Svanvik and Antrodia albobrunnea at Tennilä. $B$. grisea is fairly rare in Finland but in favourable years it is locally common especially in the north (Niemelä \& Saarenoksa 1989).

Most of the resupinate species grew on decorticated wood on the lower surface of fallen pine trunks. Botryobasidium botryosum, $B$. subcoronatum, Coniophora fusispora, Hyphoderma praetermissum and Sistotremastrum suecicum were found growing on pine bark, the latter only 
once, however. C. fusispora has been found in Finland only twice before (Kotiranta \& Larsson 1990, Kotiranta \& Saarenoksa 1993). It prefers conifer wood (Hallenberg 1985). Sceletocutis lilacina grew on spruce bark.

Wood decomposing Agaricales were found in Cortinariaceae, Crepidotaceae, Strophariaceae and Tricholomataceae, altogether 22 species. Of these four were common: Gymnopilus penetrans, Hypholoma capnoides, Mycena laevigata and Xeromphalia campanella.

Stropharia semiglobata (Strophariaceae) was the only coprophilous species, collected once on reindeer dung. Collybia tuberosa (Tricholomataceae) was the only mycophilous species and was very common, occurring at 12 sites. The fungal substrate was not identified.

Basidiolichens. Omphalina umbellifera (Tricholomataceae) was the only basidiolichen in the study. It occurred at eight sites.

\section{Notes on species}

Four species are reported from Finland for the first time: Cortinarius lux-nymphae, C. odhinnii, C. querciticus and Hebeloma bryogenes. All these are apparently widely distributed in northern Finland, and the first two were common in dry pine heaths.

The following species are northernmost records in Finland, Collybia racemosa (Keminmaa, PeP), Tricholoma imbricatum (Angeli, InL), Russula roseipes and $R$. turci (Tennilä, PeP). The general distribution of many Russula species is poorly known.

Antrodia albobrunnea, Postia lateritia, Skeletocutis lenis and S. stellae are regarded as endangered in Finland (Kotiranta \& Niemelä 1993), and S. lilacina will be included in the forthcoming list (Anttila et. al. 1995). It has been found five times before (Niemelä 1993, Anttila et al. 1995).

Except for one Rhizopogon vulgaris collection, all sporocarps were epigeous. This strenghtens the general view, that hypogeous species are rare in Fennoscandian boreal pine forests (Hansen \& Knudsen 1992). Thirty soil samples were screened annually during the study at each site.

Most sites included a number of species, five on average, which were not found at other sites. For example, the only finds of Spathularia flavida, S. rufa, Clavariadelphus sp., Clavulinopsis sp., Hygrocybe miniata, $H$. psittacina, Hygrophorus olivaceoalbus, Russula aquosa and $R$. xerampelina were made at Ainijärvi. This selection indicates a higher moisture of soil, as does the presence of Nephroma arcticum and Peltigera aphthosa and scattered Hylocomium splendens. In turn several species chracteristic of very dry sites, e.g. Coniophora fusispora, Junghuhnia luteoalba, Phellinus viticola, Pholiota heteroclita and Skeletocutis stellae, were found only at RajaJooseppi. The same was true for Naruska, which was the only site for Inocybe leptophylla, I. mixtilioides and Trechispora farinacea.

There were on an average 69 species at each site in the years 1991-1994 inclusive, but annually only 29 (range 10-40). This shows once again, that several years are required for proper monitoring of fungal species.

Several of the species are poorly known in Finland, and are described in some detail below.

\section{Tricholomataceae}

$L$.decastes and $L$. fumosum were distinguished mainly on the basis of growth habit and the coloration of the cap cuticula. $L$. fumosum grew mainly solitary and $L$. decastes was usually fasciculate. Cap cuticula were of greyish or brown shade, respectively. $L$. decastes tended to be much more robust. No differences were found in spore size or shape.

Ripartites sp. Sporocarps were produced at one site, Aska. Decomposing litter, amongst Dicranum and Pleurozium schreberi. This taxon appears to be undescribed. It resembles $R$. tricholoma but is more robust and its spores are smaller, $2-3.3 \mu \mathrm{m}$, almost globose, echinulate. It favours drier sites than $R$. tricholoma.

T. auratum coll. produced sporocarps at eight sites, characterized by open ground due to reindeer grazing. Ground vegetation is dominated by mosaic Cladonia and Dicranum vegetation except at a few sites where Pleurozium dominates. T. auratum may include two different taxa, both mycorrhizal with pine.

\section{Cortinarius subgenus Telamonia}

C. adalberti is widely ditributed but uncommon in pine forests of Lapland. In Continental Europe C. adalberti is mycorrhizal with spruce (Arnold 1993), but in the present study it was associated with pine. Cap is $1-4 \mathrm{~cm}$, hygrophanous, convex, pale clay brown to dark brown. Gills distinctly paler than cap. Stem $3-7 \times 0.2-0.5 \mathrm{~cm}$, light brown, finely fibrillose, base often pure white. Veil white. Flesh cream white to light brown. Spores (5.7)7.2-(8.0) x (3.6)-4.4-(5.0) $\mu \mathrm{m}, \mathrm{Q}=1.64$, reddish brown, warted, ellipsoid $([\mathrm{n}]=$ spores measured from 11 sporocarps). In some cases the spores were subglobose 
or broadly ellipsoid. Whether the sporocarps belong to another species cannot be decided in the present study. The characters fit well with those provided in Brandrud (1992) and Arnold (1993). C. adalberti is similar to C. abietinus and C. lux-nymphae, but the paler colour of gills than that of caps in $C$. adalberti seems to be a good distinguishing feature. The spores of $C$. adalberti are larger than those of $C$. abietinus or C. lux-nymphae.

C. armeniacus is one of the characteristic species of pine forests in Lapland. C. armeniacus is considered mycorrhizal with spruce and pine in Continental Europe (Arnold 1993); in the present study it was clearly associated with pine, but spruce cannot be excluded. Cap 2-7 cm, hygrophanous, convex, apricot to reddish brown. Gills ochre brown when young, later darker. Stem $3-8 \times 0.5-1.5 \mathrm{~cm}$, usually bulbous and not girdled, silky whitish when young, later becomes apricot brown, especially late in the season. Veil white. Flesh very light brown. Spores (7.0)-7.5-(9.0) x (4.0)-4.7-(7.8) $\mu \mathrm{m}$, $\mathrm{Q}=1.6$, usually reddish brown, slightly warted, ellipsoid $(n=18)$. The characters fit well with those provided in Brandrud (1992) and Arnold (1993), but spores are slightly smaller, $8-10 \times 5-6 \mu \mathrm{m}$ on average. There was another $C$. armeniacus-like, small-spored $(6.5 \times 4.2 \mu \mathrm{m})$ taxon at Raja-Jooseppi and Tennilä, which had Myxaciumlike cap.

C. biformis is one of the characteristic and most common species of pine forests in Lapland. In the present study it was mycorrhizal with pine. Cap is $2-6 \mathrm{~cm}$, hygrophanous, grey brown to reddish brown. Stem 5-8 $\times 0.7-1.5 \mathrm{~cm}$, white, usually slightly fibrillose, especially apex bluish. Veil sparse. Gills reddish brown when young. Flesh greyish to beige, when young with a violet coloration, without distinct taste or smell. Spores (6.2)7.6-(9.2) x (4.3)-4.9-(5.5) $\mathrm{mm}, \mathrm{Q}=1.6$, usually reddish brown, warted, ellipsoid to broadly ellipsoid $(n=50)$. The characters are in accordance with those provided by Soop (1990). This species is also typical in spruce forest (Soop 1990, Brandrud et al. 1994). Later Soop (1994) described var. robustior ined., a pine forest taxon. When dry or older, C. biformis looks like dirty C. armeniaceus. C. tortuosus (Fr.:Fr.) Fr., C. triformis Fr. ss. Moser and $C$. privignoides Henry are also very closely related and not distinguished in the present study. To conclude, what is now called $C$. biformis is perhaps heterogeneous, and requires further study.

C. cf. incisus produced sporocarps at six sites. It was mycorrhizal with pine in the present study, as it is in Continental Europe (Arnold 1993). Widely distributed but occasional. Since this taxon was not noted in the field, fresh characters are lacking. Cap is $1-3 \mathrm{~cm}$. Stem 2-4 $¥ 0.2-0.4 \mathrm{~cm}$. Spores (7.5)-8.3-(9.2) x (2.8)-3.8(4.0) $\mu \mathrm{m}, Q=2.2$, usually light brown, not distinctly warted, narrowly ellipsoid $(\mathrm{n}=5)$. Some other species with "boletoid" spores cannot be excluded for moment, viz. C. fusisporus Kühn., C. heterosporus Bres. and C. semivestitus Mos. All are mycorrhizal with pine (Arnold 1993). The present taxon more closely resembles C. fusisporus than C. incisus of Arnold (1993). C. fusisporus (Arnold 1993) and C. incisus (Brandrud 1992) seem to be very similar, however. Spores of $C$. heterosporus seem not to be identical with the present taxon, and spores of $C$. semivestitus are bigger (9-11 x 4-4.5 $\mu \mathrm{m})$ (Arnold 1993). The taxon also resembled C. lux-nymphae.
C. cf. laetus produced sporocarps at one site, Muonio. Cap is $1.5 \mathrm{~cm}$, dry, milk brown. Stem $3 \times 0.2 \mathrm{~cm}$, concolorous with the cap. Velum brown, sparse. Spores (7.2)-8.2-(9.2) x (4.0)-5.4-(4.0) $\mu \mathrm{m}, \mathrm{Q}=1.52$, brown, warted, ellipsoid $(\mathrm{n}=1)$.

C. lux-nymphae (Fig. 3) is one of the characteristic species of lichen-rich pine forests in southern Lapland. It was especially abundant at Tennilä. Cap is $1-4 \mathrm{~cm}$, hygrophanous, convex to plane, pale brown (when dry) to dark brown (when moist), scaly especially when young, as in C. paleaceus. Gills brown to dark brown. Stem 1$2 \times 0.2-0.4 \mathrm{~cm}$, light brown to brown. Veil white. Flesh light brown to ochre when dry. Spores (5.4)-6.3-(7.0) $\mathrm{x}$ (2.9)-3.4-(4.1) $\mu \mathrm{m}, \mathrm{Q}=1.88$, usually very light brown, almost smooth, narrowly ellipsoid to ellipsoid $(n=42)$. The characters fit well with those provided by Brandrud et al. (1994). A small Telamonia not reported in Finland before, very common in northern oligotrophic pine forests. A characteristic feature facilitating identification is the small and narrow spores. A closely related C. abietinus is mycorrhizal with spruce in Continental Europe (Arnold 1993).

C. odhinnii is one of the characteristic species of pine forests in Lapland. Mycorrhizal with pine in the present study. Cap is $1-4 \mathrm{~cm}$, campanulate to convex, when fresh bright brown to orange brown, darkening when dry or dried. Gills paler than cap. Stem $4-8 \times 0.4-0.8$ $\mathrm{cm}$, first white, but turns to golden yellow and yellowish brown. Veil white. Flesh yellowish to yellowish brown. Spores (6.2)-7.5-(9.5) x (3.2)-4.2-(5.4) $\mu \mathrm{m}, \mathrm{Q}=1.79$, usually reddish brown, slightly warted, ellipsoid $(n=56)$. These characters fit fairly well with those provided by Brandrud et al. (1992), although variation in spore size is greater. It was sometimes difficult to judge between dried C. lux-nymphae and C. odhinnii, if not supported by field notes.

Fig. 3. Cortinarius lux-nymphae, mature sprocarps after rain (14.8.1993, Keminmaa).

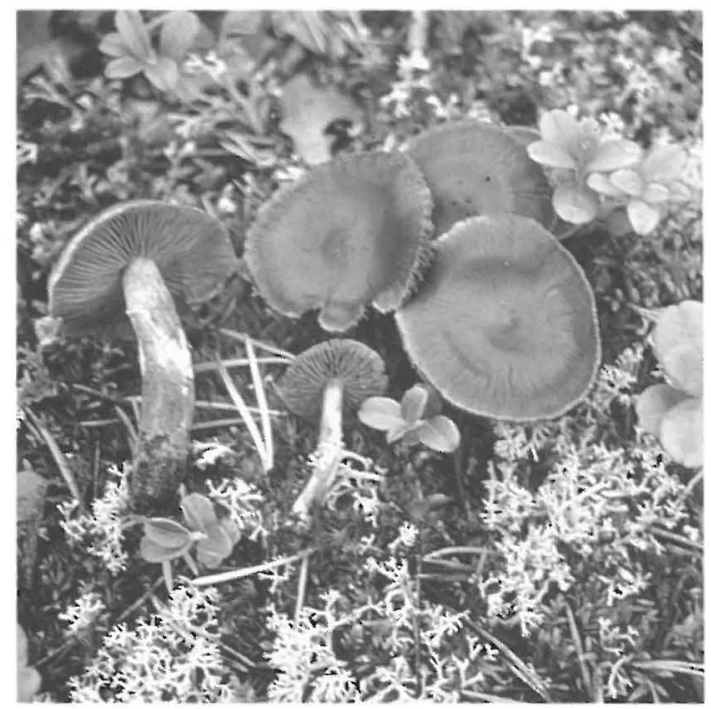


C. paragaudis subsp. paragaudis resembled $C$. armillatus, and is regarded as rare in Finland (Brandrud 1992). In northern Finland it is widespread and not rare, however. At some sites it occurred each year. Spores (5.4)-6.7-(8.0) x (4.4)-5.3-(5.6) $\mu \mathrm{m}, \mathrm{Q}=1.26$, light brown to brown, and slightly warted to warted, broadly ellipsoid to subglobose $(n=15)$. These characters fit well the descriptions in Brandrud et al. (1992) and Arnold (1993).

\section{Cortinarius subgenus Phlegmacium}

C. leucophanes is a characteristic species of dry pine forests in Lapland. Cap is $3-6 \mathrm{~cm}$, viscid, not hygrophanous, milk brown to ivory white. Stem $4-7 \times 0.5-0.9 \mathrm{~cm}$, white and fibrillose when young, brown yellow when older. Velum white, abundant. Gills crowded, bright brown. Flesh white to brownish, without distinctive taste or smell Spores (5.0)-5.7-(6.0) x (3.1)-3.4-(4.0) $\mu \mathrm{m}, \mathrm{Q}=1.67$, usually light brown, not distinctly warted, ellipsoid $(n=20)$. The characters accord perfectly with those provided by Soop (1991). A recognizable species growing in most oligotrophic lichen heaths.

\section{Cortinarius subgenus Sericeocybe}

C. ochrophyllus is mycorrhizal with birch, as suggested by Moser (1983). C. ochrophyllus resembles C. anomalus (Soop 1986), but its gills are yellow brown, while those of $C$. anomalus are greyish brown with a violet shade. Cap of C. ochrophyllus cap remains milk brown, relatively bright ochraceous brown to greyish brown, stem is white, often with remnants of veil. Both the cap and stem are fibrillose. An olive shade appears in the light brown bottom colour of the dry cap and stem. Spores (6.2)-6.8-(8.7) $\times$ (4.3)-5.1-(6.2) $\mu \mathrm{m}, \mathrm{Q}=1.33$, usually reddish brown and distinctly warted, broadly ellipsoid to subglobose $(n=7)$. The spore size is somewhat smaller than Moser (1983) reported. One collection (Kirakkajärvi) had smaller spores (not included in above), (4.7)-5.6-(6.2) x (3.9)-4.1-(4.8) $\mu \mathrm{m}$, and the stem was whiter and fibrillose.

C. querciticus was mycorrhizal with pine in the present study. Very local, but produced sporocarps each year at one site, Lokka. Cap is $4-10 \mathrm{~cm}$, dry, brownish with violet shade, silky fibrillose. Stem is $1.5-2.5 \mathrm{~cm}$, bulbous, white fibrillose with strong violet coloration especially when young. Gills crowded. Flesh violet. Spores (7.0)-7.9-(8.3) x (4.3)-4.9-(5.8) $\mu \mathrm{m}, \mathrm{Q}=1.61$, usually brown, distinctly warted and ellipsoid $(n=7)$. These characters fit well with descriptions in Soop (1993ab, as C. pseudomalachius Reumaux) and Brandrud et al. (1994).

Cortinarius sp. sporocarps were produced at two sites in 1992. This taxon was mycorrhizal with pine. Cap is $2-4 \mathrm{~cm}$, dry. When dried, showing an olive shade in both stem and cap. Spores (6.5)-7.4-(9.1) x (4.1)-4.5(5.4) $\mu \mathrm{m}, \mathrm{Q}=1.64$, brown, warted, ellipsoid $(\mathrm{n}=5)$.

\section{Cortinarius subgenus Leprocybe}

C. raphanoides is one of the characteristic species of pine forests with birch as undergrowth, and $C$. raphanoides clearly was mycorrhizal with birch. The yield was highest at sites with mosses dominating the ground vegetation (especially Kemijärvi). Cap is $1.5-6 \mathrm{~cm}$, hygro- phanous, surface silky, smooth, greyish brown to olive brown. In a few cases the coloration of cap was lighter, as Høiland (1980) has noted. Stem 5-8 x 0.7-1.0 cm, pale, usually slightly fibrillose, especially apex bluish. Veil grey brown. Gills olivaceous, with a cinnamon shade sometimes. At one site, Kemijärvi, it formed a fairy ring. Spores (7.0)-7.9-(8.0) x (4.0)-4.8-(5.0) $\mu \mathrm{m}, \mathrm{Q}=1.65$, ellipsoid, warted $(n=10)$. Spore size is somewhat smaller than that reported by Moser (1983), but agrees with that reported by Høiland (1980).

\section{Hebeloma}

$H$. birrus produced sporocarps only at Muonio, where the soil is silty and the ground vegetation dominated by Pleurozium schreberi, Dicranum fuscescens and D. polysetum. Mycorrhizal with pine in the present study. Sect. Scabrispora or Birri (Vesterholt 1994, in. let.). Macroscopic details are presented in Vesterholt (1994). Spores were amygdaloid to ellipsoid, rugose, brown (7.7)-8.6(9.2) x (4.6)-5.3-(6.2) $\mu \mathrm{m}$, and cheilocystidia were short and cylindrical $(n=1)$, in agreement with Vesterholt's description (1994). A poorly known species in Finland, but it seems to be fairly common in southern Sweden (Stridvall \& Stridvall 1994), in moist places and at burntover ground. In Denmark it is fairly rare (Vesterholt 1994).

H. bryogenes (Fig. 4) produced sporocarps at three sites, which were dominated by Pleurozium schreberi, Dicranum fuscescens and D. polysetum. Mycorrhizal at least with pine. Sect. Denudata. Macroscopic details are presented in Vesterholt (1994). Spores slightly rugose, amygdaloid, brown (9.2)-10.1-(11.5) x (5.0)-5.8-(6.2) $\mathrm{\mu m}$ $(\mathrm{n}=5)$. Cheilocystidia cylindrical, narrow $(4-6.5 \mu \mathrm{m})$ and long, up to $80 \mu \mathrm{m}(\mathrm{n}=5)$. These characters agree with that presented in Vesterholt (1993). It has been reported to be mycorrhizal with pine and spruce, especially at moist sites, e.g. amongst Sphagnum (Vesterholt 1993). Very common in Scandinavia (Vesterholt 1993, Stridvall \& Stridvall 1994).

Both $H$. bryogenes and $H$. leucosarx were earlier named as $H$. longicaudum. H. longicaudum is considered a nomen dubium, however (Vesterholt 1993), and thus replaced by $H$. leucosarx (Vesterholt 1992). Both have been also confused with $H$. crustuliniforme (Bull.) Quél. H. bryogenes is widely distributed in mossy pine forests of northern Finland.

H. leucosarx produced sporocarps at five sites, which were dominated by Pleurozium schreberi. May be mycorrhizal with spruce, pine or birch. Birch and spruce occurred at three sites and pine at all five. Sect. Denudata. Macroscopic details are presented in Vesterholt (1992). Spores rugose, amygdaloid, brown (9.2)-10.2-(11.0) $x$ (5.4)-6.3-(7.0) $\mu \mathrm{m}$, cheilocystidia cylindric, 7-9 $\mu \mathrm{m}$ broad $(\mathrm{n}=8)$. These characters fit well with those presented in Vesterholt $(1992,1993)$. Microscopically H. leucosarx can be distinguished from $H$. bryogenes by broader cheilocystidia (Vesterholt 1993). H. leucosarx is common in Denmark (Vesterholt 1992) and southern Sweden (Stridvall \& Stridvall 1994) and is widely distributed in mossy pine forests of northern Finland.

Hebeloma sp. Spores rugose, amygdaloid, light brown (11)-11.9-(13.0) × (6.2)-6.5-(7.0), cheilocystidia $7 \mu \mathrm{m}$ broad $(n=1)$. Resembles $H$. leucosarx. 
Inocybe

Inocybe is fairly poorly known in oligotrophic pine forests in northern Finland, and only one of the species recognized here was included in the key to Nordic Macromycetes (Printz 1992). Macro- and microscopic details are provided in Vauras (1992).

I. jacobi produced sporocarps at one site, Muonio. Like $H$. birrus, it prefers silty soil (Vauras 1992). Evidently mycorrhizal with pine. Other species in the section prefer moist deciduous or spruce forests (Vauras 1992).

I. lacera produced sporocarps at three sites, which were dominated by various Cladonia species. Var. heterosperma grew in Ainijärvi together with var. lacera. Mycorrhizal with pine.

I. leptophylla produced sporocarps at two sites. In 1993 I. leptophylla was especially abundant at Naruska Both sites were dominated by various Cladonia species. Mycorrhizal with pine.

I. mixtiloides produced sporocarps at one site, Naruska, which is dominated by Cladonia spp. and Dicranum fuscescens. Mycorrhizal with pine.

I. soluta produced sporocarps at nine sites, which were dominated by various Cladonia species. Mycorrhizal with pine. Mostly occasional, but occurred each year at three sites. The most common Inocybe in the present study. Vauras (1992) considers I. lacera to be the most common Inocybe on the dry sandy soils of Finland (Vauras 1992), but also $I$. subcarpta was more common than $I$. lacera in the present study.

I. subcarpta produced sporocarps at four sites. The sporocarp yield was highest at the sites dominated by mosses, especially in Kemijärvi. Mycorrhizal with pine. In Nordic Macromycetes (Printz 1992) this species is presented as I. boltonii, but the valid name is $I$. subcarpta (Vauras 1992).

\section{Russula}

$R$ sp. in sectio Russula is an undescribed species resembling $R$. emetica but more common than it in northern Finland (J. Ruotsalainen, pers. comm.). It produced sporocarps at four sites, all of them characterized by mosaic lichen and moss vegetation. It is much more common than $R$. emetica in Finland (J. Ruotsalainen, pers. comm.). Mycorrhizal host is pine or birch or both. Spruce occurred at two sites.

\section{The yield}

The sporocarp yield varied markedly from one year to the next. The best season was 1993 with $9685 \mathrm{~g} \mathrm{dw} \mathrm{ha}^{-1}$, and the poorest 1994 with $1115 \mathrm{~g} \mathrm{dw} \mathrm{ha}^{-1}$ (Table 3). The summer of 1994 was very dry in most parts of northern Finland, and perhaps this influenced the yield. Other years were fairly similar in temperature and rainfall. The annual yield was $4600 \mathrm{~g} \mathrm{dw}$ $\mathrm{ha}^{-1}$ on average, and the range $40-18180 \mathrm{~g} \mathrm{dw}$ $\mathrm{ha}^{-1}$. Since the sporocarps were collected only

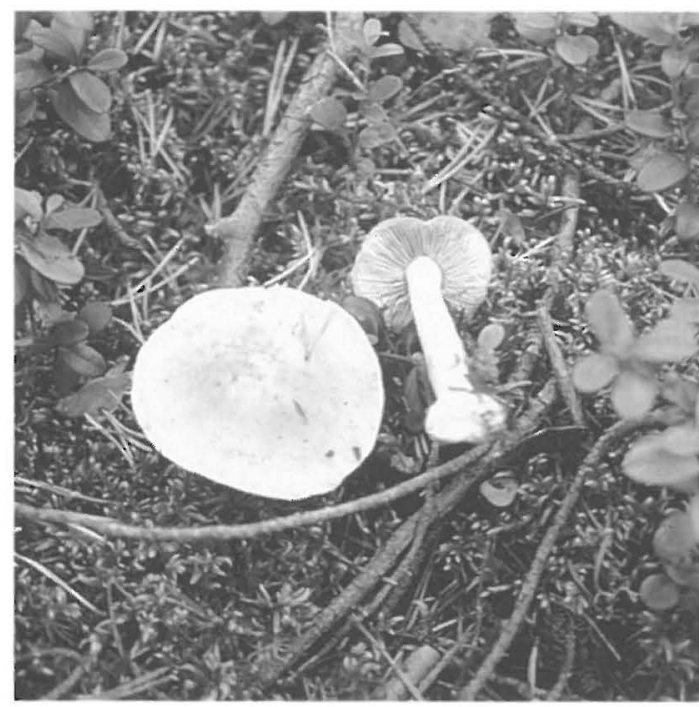

Fig. 4. Hebeloma bryogenes (11.9.1993, Keminmaa).

once or twice, the total yield is certainly underestimated. On the basis of Paulus (1995), who followed the yield in northern Lapland for a longer period in 1992 and 1993 we estimate that yield in our study covers $75-80 \%$ of the total yield. The proportion may be lower in the southern than northern parts of our study area.

Not all species were abundant in 1993 (Table 3). The maximum yield in this year was mainly due to the four most common species - Lactarius rufus (2495 g dw ha ${ }^{-1)}$, Russula decolorans $\left(1095 \mathrm{~g}\right.$ dw ha ${ }^{-1)}, R$. paludosa $(785 \mathrm{~g}$ $\mathrm{dw} \mathrm{ha}^{-1)}$, and Suillus variegatus (3080 $\mathrm{g} \mathrm{dw}$ ha 1) - which were responsible for $75 \%$ of the total yield (Table 3). The proportion of mycorrhizal fungi was $97.6 \%$ of the total yield (93.7-98.4\%). The yield was of the same magnitude as in earlier studies carried out in oligotrophic dryish pine forests of northern Fennoscandia (Ohenoja \& Koistinen 1984, Mehus 1986, Ohenoja 1993), which further supports our contention, that the yield we observed covers most of the total yield. The species reported in these earlier studies were mostly the same as in our study, and as regards pine forests the yield was highest in dryish mossy sites. The total yield in oligotrophic dry pine forests over 40 years of 
age in the province of Lapland was estimated to be 13 million $\mathrm{kg}$ dw (about 130 million $\mathrm{kg}$ $\mathrm{fw})$. An earlier estimate with all forest types included is close to this: 300 million $\mathrm{kg}$ fw (Kujala et al. 1987).

Agaricales, Boletales and Russulales dominated the yield. Agaricales was dominated by Cortinariaceae and Boletales by Gomphidiaceae. Four genera produced $85 \%$ of the fungal yield; Cortinarius accounted for 20\%, Lactarius for 26\%, Russula for 15\% and Suillus for $27 \%$. The two most productive species, Lactarius rufus and Suillus variegatus, accounted for $50 \%$ of the yield (Tables 3 and 5). Cortinarius biformis, C. brunneus and C. semisanguineus were the most productive species in the genus Cortinarius and $R$. decolorans and $R$. paludosa in Russula.

The yield was highest at the two southernmost study sites, which are located in the middle boreal zone (Fig. 1). The yield was lowest in eastern Lapland. The differences were also related to quality of vegetation, the yield being lower at dry lichen-rich sites than at mossy sites. These and other ecological aspects will be discussed in forthcoming articles.

\section{Edible species}

Species which are commonly consumed in Finland (for example edible Cortinarius were excluded) and which are of relevant size, were considered as edible fungi. The average annual yield of edible species was $3160 \mathrm{~g} \mathrm{dw} \mathrm{ha}^{-}$ ${ }^{1}$, nearly $70 \%$ of the measured total yield ( $\mathrm{Ta}-$ ble 5). Between 1976 and 1978 the proportion was calculated to be $68 \%$ (Ohenoja \& Koistinen 1984), with the edible species mostly the same as in the present study. Only one edible decomposer, Hypholoma capnoides, produced notable yields in the present study. Its average annual yield was $23 \mathrm{~g} \mathrm{dw} \mathrm{ha}^{-1}$. In all, 27 edible mycorrhizal species produced sporocarps, but the annual yield was over $10 \mathrm{~g} \mathrm{dw} \mathrm{ha}{ }^{-1}$ for only 12 of these. Other species may be locally of some value at certain years, however. Lactarius rufus produced $35 \%$ of the total edible yield, Suillus variegatus $38 \%$ and four species of the genus Russula $21 \%$. As regards commercial picking of fungi in northern Finland, only $L$. rufus, $R$. decolorans, $R$. paludosa, $R$. vinosa and $S$. variegatus can be regarded as of any importance. Edible fungi and their yields are listed in Table 5.

Table 5. The most common edible fungal species, their mean annual yield $\left(\mathrm{g} \mathrm{dw} h \mathrm{~h}^{-1}\right)$, their proportion of the edible species and of all species in 1991-1994, estimate in oligotrophic pine forests over 40 years old in the province of Lapland. Only species with average annual yield over $10 \mathrm{~g} \mathrm{dw} \mathrm{ha}^{-1}$ are included.

\begin{tabular}{|c|c|c|c|c|}
\hline Species & $\begin{array}{c}\text { mean } \\
\mathrm{g} \mathrm{dw} \mathrm{ha} \mathrm{a}^{-1} \\
\end{array}$ & $\begin{array}{c}\text { proportion } \\
\text { of edible }\end{array}$ & $\begin{array}{l}\text { proportion } \\
\text { of total }\end{array}$ & $\begin{array}{c}\text { Lapland total } \\
\mathrm{kg} \mathrm{dw}\end{array}$ \\
\hline Hypholoma capnoides & 23 & 0.8 & 0.5 & 63700 \\
\hline Lactarius mammosus & 24 & 0.8 & 0.5 & 66500 \\
\hline L. rufus & 1100 & 34.5 & 23.9 & 3046700 \\
\hline Leccinum scabrum & 17 & 0.6 & 0.4 & 47100 \\
\hline L. versipelle & 31 & 1.1 & 0.7 & 85900 \\
\hline L. vulpinum & 17 & 0.6 & 0.4 & 47100 \\
\hline Russula claroflava & 12 & 0.5 & 0.3 & 33200 \\
\hline R. decolorans & 340 & 10.7 & 7.4 & 941700 \\
\hline R. paludosa & 250 & 7.9 & 5.4 & 692400 \\
\hline$R$. vinosa & 73 & 2.4 & 1.6 & 202200 \\
\hline Suillus bovinus & 62 & 2.0 & 1.3 & 171700 \\
\hline S. variegatus & 1200 & 37.6 & 26.1 & 3323700 \\
\hline Tricholoma auratum & 14 & 0.5 & 0.3 & 38800 \\
\hline Total & 3163 & 100.0 & 68.8 & 8760700 \\
\hline
\end{tabular}


Acknowledgements. Jukka Vauras (Finland), Jan Vesterholt (Denmark) and Juhani Ruotsalainen (Finland) provided most valuable help in identifying Inocybe, Hebeloma and Russula samples, respectively. Stig Jacobsson (Sweden) helped with some Pholiota samples, and discovered Galerina camerina among these. Klaus Høiland (Norway) and Karl Soop (Sweden) helped with some Cortinarius, Egil and Kati Bendiksen (Norway) determined Cortinarius adalbertii, and Heikki Kotiranta (Finland) checked most polyporoid and corticioid collections. Our warm thanks to all. Kauko Salo and an anonymous reviewer provided useful comments on the manuscript. The study was supported by the Ministry of Agriculture and Forestry.

\section{References}

Aarne, M. (ed.) 1994: Yearbook of Forest Statistics 1993-94. - 348 pp. Gummerus. Jyväskylä.

Agerer, R. 1993: Ectomycorrhizae of Hydnellum peckii on Norway spruce and their chlamydospores. Mycologia 85:74-83.

Ahti, T., Hämet-Ahti, L. \& Jalas, J. 1968: Vegetation zones and their sections in northwestern Europe. Ann. Bot. Fennici 5:169-211.

Anttila, S., Uimonen, J. \& Vehmaa, P. 1995: Käävät aarnimetsien ilmentäjinä Kainuun kuusikoissa (Summary: Polypores on decomposing spruce trunks in old forests of eastern Central Finland). - Lutukka 11:42-48.

Arnold, N. 1993: Morphologisch-anatomische und chemische Untersuchungen an der Untergattung Telamonia (Cortinarius, Agaricales). - Libri Bot. 7:1213.

Brandrud, T. E. 1992: Subgen. Telamonia (Fr.) Loudon. In: Hansen, L. \& Knudsen, H. (eds.), Nordic Macromycetes Vol. 2:299-306. - Nordsvamp, Copenhagen.

Brandrud, T. E., Lindström, H., Marklund, H., Melot, J. \& Muskos, S. 1992: Cortinarius Flora, Photographica, vol. 2 (English version). - Cortinarius HB, Matfors.

Brandrud, T. E., Lindström, H., Marklund, H., Melot, J. \& Muskos, S. 1994: Cortinarius Flora, Photographica, del. 3 (Swedish version). - Cortinarius HB, Matfors.

Currah, R. S. \& Zelmer, C. 1992: A key and notes for the genera of fungi mycorrhizal with orchids and a new species in the genus Epulorhiza. - Rep. Tottori Mycol. Inst. 30:43-59.

Danielson, R. 1984: Ectomycorrhizal association in jack pine stands in northern America. - Canadian J. Bot. 62:932-939.

Entry, J. A., Rose, C. L. \& Cromack, K., Jr. 1991: Litter decomposition and nutrient release in ectomycorrhizal mat soils of a Douglas fir ecosystem. - Soil Biol. Biochem. 23:285-290.

Eriksson, J., Hjortstam, K. \& Ryvarden, L. 1978: The Corticiaceae of North Europe 5. Mycoaciella to Phanerochaete. - Pp. 889-1047. Fungiflora, Oslo.

Eriksson, J., Hjortstam, K. \& Ryvarden, L. 1981: The Corticiaceae of North Europe 6. Phlebia to Sarcodontia. - Pp. 1051-1276. Fungiflora, Oslo.
Eriksson, J., Hjortstam, K. \& Ryvarden, L. 1984: The Corticiaceae of North Europe 7. Schizopora to Suillosporium. - Pp. 1281-1449. Fungiflora, Oslo.

Eriksson, J. \& Ryvarden, L. 1973: The Corticiaceae of North Europe 2. Aleurodiscus to Conferto-basidium. - Pp. 59-261. Fungiflora, Oslo.

Eriksson, J. \& Ryvarden, L. 1975: The Corticiaceae of North Europe 3. Coronicium to Hyphoderma. - Pp. 287-546. Fungiflora, Oslo.

Eriksson, J. \& Ryvarden, L. 1976: The Corticiaceae of North Europe 4. Hyphodermella to Mycoacia. - Pp. 549-886. Fungiflora, Oslo.

Gilbertson, R. L. \& Ryvarden, L. 1986: North American Polypores 1. Abortiporus to Lindt-neria. Pp. 1-433. Fungiflora, Oslo.

Hallenberg, N. 1985: The Lachnocladiaceae and Coniophoraceae of North Europe. - 96 pp. Fungiflora, Oslo.

Hansen, L. \& Knudsen, H. (eds.) 1992: Nordic Macromycetes 2. - 474 pp. Nordsvamp, Copenhagen.

Hansen, L. \& Knudsen, H. (eds.) 1995: Nordic Macromycetes 1. - Nordsvamp, Copenhagen (in press).

Hintikka, V. 1988: On the macromycete flora in oligotrophic pine forests of different ages in South Finland. - Acta Bot. Fennica 136:89-94.

Hjortstam, K., Larsson, K.-H. \& Ryvarden, L. 1988: The Corticiaceae of North Europe 8. Thanatephorus to Ypsilonidium. - Pp. 1450-1631. Fungiflora, Oslo.

Høiland, K. 1980: Cortinarius subgenus Leprocybe in Norway. - Norwegian J. Bot. 27:101-126.

Høiland, K. 1984: Cortinarius subgenus Dermocybe. Opera Bot. 71:1-113.

Jülich, W. 1984: Die Nichtblätterpilze, Gallertpilze und Bauchpilze (Aphyllophorales, Heterobasiodiomycetes, Gastromycetes). In: Gams, H. (ed.), Kleine Kryptogamenflora 2b(1), Basidiomyceten 1. - 626 pp. Gustav Fischer Verlag, Stuttgart \& New York.

Kalela, A. 1961: Waldvegetationszonen Finnlands und ihre klimatische Paralleltypen. - Arch. Soc. Zool. Bot. Fennicae Vanamo 16(Suppl.):65-83.

Kolkki, O. 1966: Taulukoita ja karttoja Suomen lämpötilasta kaudelta 1931-1960. (Summary: Tables and maps of temperature in Finland during 1931-1960). Suppl. Meteorol. Yearb. of Finland 65(1a):1-42.

Kotiranta, H. \& Larsson, K.-H. 1990: New or little collected corticolous fungi from Finland (Aphyllophorales, Basidiomycetes). - Windahlia 18:1-14.

Kotiranta, H. \& Niemelä, T. 1981: Composition of the polypore communities of four forest areas in southern Central Finland. - Karstenia 21:31-48.

Kotiranta, H. \& Niemelä, T. 1993: Uhanalaiset käävät Suomessa. - Vesi- ja ympäristöhallinnon julkaisuja 17:1-116.

Kotiranta, H. \& Saarenoksa, R. 1993: Rare Finnish Aphyllophorales (Basidiomycetes) plus two new combinations in Efibula. - Ann. Bot. Fennici 30:211-249.

Kujala, M., Kynsilehto, K., Ohenoja, E., Saastamoinen, O. \& Sepponen, P. 1987: Lapin läänin luonnonmarja- ja sienivarat, niiden satoarviot, hyödyntäminen ja sivuansiollinen merkitys. -60 p. Polar-Marsi 86, a report.

Maas Geesteranus, R. A. 1975: Die terrestrischen Stachelpilze Europas. - Verh. Konigl. Nederl. Akad. Wetensch. Afd. Natuurk. 65:1-160. 
Mehus, H. 1986: Fruit body production of macrofungi in some North Norwegian forest types. - Nordic J. Bot. 6:679-702.

Molina, R., Massicotte, H. \& Trappe, J. M. 1992: Specificity phenomena in mycorrhizal symbioses: community-ecological consequences and practical implications. In Allen, M. F. (ed.), Mycorrhizal functioning. An integrative plant-fungal process:357423. - Chapman \& Hall, New York.

Moser, M. 1983: Cortinarius raphanoides Pers.:Fr. and related species. - Cryptogamie, Mycologie 4:41-50.

Niemelä, T. 1993: Suomen kääpien määritysopas, 7. painos. - Helsingin yliopiston kasvitieteen laitoksen monisteita 133:1-124.

Niemelä, T. Kotiranta, H. 1993: Orakkaitten määritysopas, 2. painos. - Helsingin yliopiston kasvitieteen laitoksen monisteita 134:1-55.

Niemelä, T. \& Saarenoksa, R. 1989: On Fennoscandian polypores 10. Boletopsis leucomelaena and B. grisea described and illustrated. - Karstenia 29:12-28.

Ohenoja, E. 1993: Effect of weather conditions on the larger fungi at different forest sites in northern Finland in 1976-1988. - Acta Univ. Oul. A 243:1-69.

Ohenoja, E. 1995: Effect of winter conditions on the fruit body production of larger fungi. - Symb. Bot. Upsalienses 30(3):163-168..

Ohenoja, E. \& Koistinen, R. 1984: Fruit body production of larger fungi in Finland 2. Edible fungi in northern Finland 1976-1978. - Ann. Bot. Fennici $21: 357-366$

Ohenoja, E. \& Väre, H. 1993: Larger fungi of the Suvanto area along the river Kitinen, Central Lapland. - Memoranda Soc. Fauna Flora Fennica 69:87-96.

Ojansuu, R. \& Henttonen, H. 1983: Kuukauden keskilämpötilan, lämpösumman ja sademäärän paikallisten arvojen johtaminen ilmatieteenlaitoksen mittaustiedoista (Summary: Estimation of local values of monthly mean temperatures, effective temperature sum and precipitation sum from the measurements made by the Finnish Meteorol-ogical Institute). Silva Fennica 17:143-160.

Paulus, A. 1995: Metsänhakkuun vaikutuksesta sieniyhteisöihin. - MSc. thesis, Department of Biol-ogy, University of Oulu.

Printz, P. 1992: Inocybe (Fr.) Fr. In: Hansen, L. \& Knudsen, H. (eds.), Nordic Macromycetes Vol. 2:321-330. - Nordsvamp, Copenhagen.

Renvall, P. 1995: Community structure and dynamics of wood-rotting Basidiomycetes on decomposing conifer trunks in northern Finland. - Karstenia 36:151.

Renvall, P., Renvall, T. \& Niemelä, T. 1991: Basidiomycetes at the timberline in Lapland 2. An annotated checklist of the polypores of north-eastern Finland. - Karstenia 31:13-28.

Salo, K. 1993: The composition and structure of macrofungus communities in boreal upland type forests and peatlands in North Karelia, Finland. Karstenia 33:61-99.

Soop, K. 1986: Några observationer av spindelskivlingar inom Sericeocybe grupp Anomali. - Jordstjärnan 7:5-10.

Soop, K. 1990: Observationer om några intressanta Cortinarius-arter. - Agarica 10/11:30-43.

Soop, K. 1991: Ovanliga Cortinarius-arter - en bildserie, del V. - Jordstjärnan 12:15-18.

Soop, K. 1993a: On Cortinarius in boreal pine forests. Agarica 12(21):101-116.

Soop, K. 1993b. Ovanliga Cortinarius-arter - en bildserie, del VII. - Jordstjärnan 14:44-49.

Soop, K. 1994: En ny titt på spindlingar inom gruppen Smör \& Bröd. - Jordstjärnan 15:12-19.

Stridvall, L. \& Stridvall, A. 1994: Intressanta skivlingfynd från fyrstadsområdet I. Conocybe, Galerina, Hebeloma. - Jordstjärnan 15:43-64.

Taylor, A. S. F. \& Alexander, I. J. 1991: Ectomycorrhizal synthesis with Tylospora fibrillosa, a member of the Corticiaceae. - Mycorrhizal Res. 95:381-384.

Trappe, J. M. 1962: Fungus associates of ectotrophic mycorrhizae. - Bot. Rev. 28:538-606.

Trappe, J. M. 1971: Mycorrhiza-forming ascomycetes. In: Hacskaylo, E. (ed.) Mycorrhizae. U.S. Dept. Agric., Forest Serv. Misc. Publ. 1189:19-37.

Tuhkanen, S. 1984: A circumboreal system of climaticphytogeographical regions. - Acta Bot. Fennica 127:1-50.

Väre, H. 1989: The mycorrhizal condition of weakened Scots pine saplings on ploughed sites in northern Finland. - Canad. J. For. Res. 19:341-346.

Väre, H., Ohtonen, R. \& Oksanen, J. 1995: Effects of reindeer grazing on understorey vegetation in dry Pinus sylvestris forests. - J. Veg. Sci. 6:523-530.

Vauras, J. 1992: Suomen risakkaiden (Inocybe, Agaricales) systematiikasta ja ekologiasta. - 145 p. Lic. Phil. Thesis, University of Turku, Department of Biology.

Vesterholt, J. 1992: Almindelig tåreblad (Hebeloma crustuliniforme) og dens almindelige dobbeltgængere. - Svampe 25:15-22.

Vesterholt, J. 1993: Taxonomic notes on Hebeloma (Agaricales, Cortinariaceae). - Windahlia 20:55-61.

Vesterholt, J. 1994: De rodslående og sødtduftende arter af tåreblad (Hebeloma). - Svampe 29:13-27.

Received on 12 January 1995 